\title{
Recurrence of a right ventricular hemangioma
}

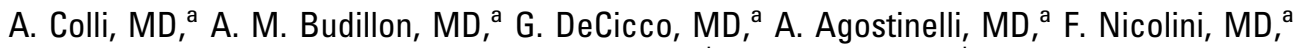 \\ D. Tzialtas, MD, ${ }^{a}$ G. Zoffoli, MD, ${ }^{a}$ D. Corradi, MD, ${ }^{b}$ R. Maestri, PhD, ${ }^{b}$ C. Beghi, MD, ${ }^{a}$ and \\ T. Gherli, MD, ${ }^{\text {a Parma, Italy }}$
}

$\mathrm{T}$ he majority of cardiac tumors are benign, with only $25 \%$ to $30 \%$ being histologically malignant. Only $0.8 \%$ to $5 \%$ of all benign primary cardiac tumors are hemangiomas. ${ }^{1,2}$

Cardiac hemangiomas are vascular tumors, composed of capillaries or cavernous vascular channels. ${ }^{3}$ Patients usually have a variety of symptoms depending on location and extension of the tumor. Diagnosis and management of these tumors are difficult and delayed and require multiple investigations.

We report a case of late recurrence of a cardiac hemangioma of the right ventricle from surgical treatment at our institution 10 years previously.

\section{Clinical Summary}

A 69-year-old man who had resection of a hemangioma of the right ventricle 10 years before was readmitted to our institution because of echocardiographic findings of an ovular mass $(1.6 \times$ $2.7 \mathrm{~cm}$ ) under the septal leaflet of the tricuspid valve (Figure 1, A). The neoformation determined mild tricuspid regurgitation. The patient had been pharmacologically treated 6 months before for an episode of supraventricular paroxysmal tachycardia. Until then, no pathologic findings had been reported by the previously scheduled echocardiograms.

At readmission, physical examination showed no abnormalities. Coronary angiograms revealed normal coronary arteries and a contrast blush arising from the distribution territory of the septal branches of the left anterior descending coronary artery.

An intraoperative transesophageal echocardiogram confirmed the presence of an infiltrating septal mass under the tricuspid valve causing mild tricuspid regurgitation. Cardiopulmonary bypass was instituted with femoral artery and bicaval cannulation, and cardioplegic arrest was performed with normothermic blood cardioplegia. Through a right atriotomy and the tricuspid valve, the interventricular septum was approached and showed a brownish, not well-encapsulated, smooth mass with an approximate size of 3 $\times 2 \times 1 \mathrm{~cm}$. The mass had a large implant base attached to the interventricular septum and appeared to have the same location of

\footnotetext{
From the Departments of Cardiac Surgery ${ }^{\mathrm{a}}$ and Pathology, ${ }^{\mathrm{b}}$ University of Parma, Parma, Italy.

Received for publication Dec 29, 2002; accepted for publication Jan 7, 2003.

Address for reprints: A. Colli, MD, Cattedra e Divisione di Cardiochirurgia, Università degli Studi, Via A. Gramsci 14, 43100 Parma, Italy (E-mail: colli.andrea@libero.it).

J Thorac Cardiovasc Surg 2003;126:881-3

Copyright (C) 2003 by The American Association for Thoracic Surgery

$0022-5223 / 2003 \$ 30.00+0$

doi:10.1016/S0022-5223(03)00720-7
}

the first presentation. The tumor was excised and no septal perforation occurred. Postoperative echocardiography showed the absence of a ventricular septal defect, a mild tricuspid regurgitation, and normal heart con-

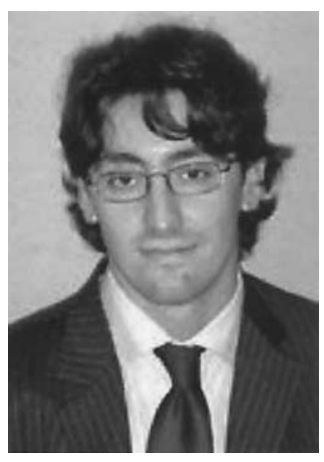

Dr Colli tractility (Figure 1, B). The postoperative course was uneventful and the patient was discharged home on the seventh postoperative day.

On morphologic and histologic examination, both neoplasms showed the same characteristics. Grossly, they were brownish,
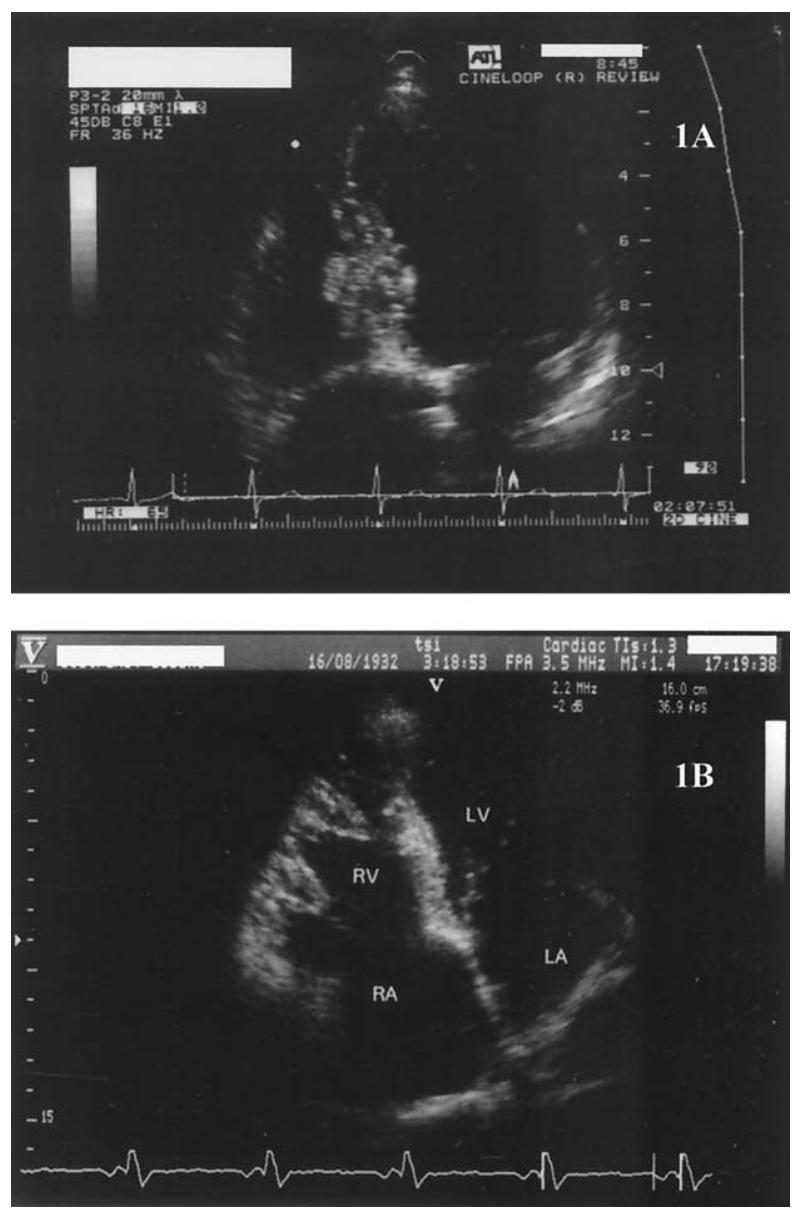

Figure 1. A, Preoperative transthoracic echocardiogram showing the right ventricular hemangioma. B, Postoperative transthoracic echocardiogram. 


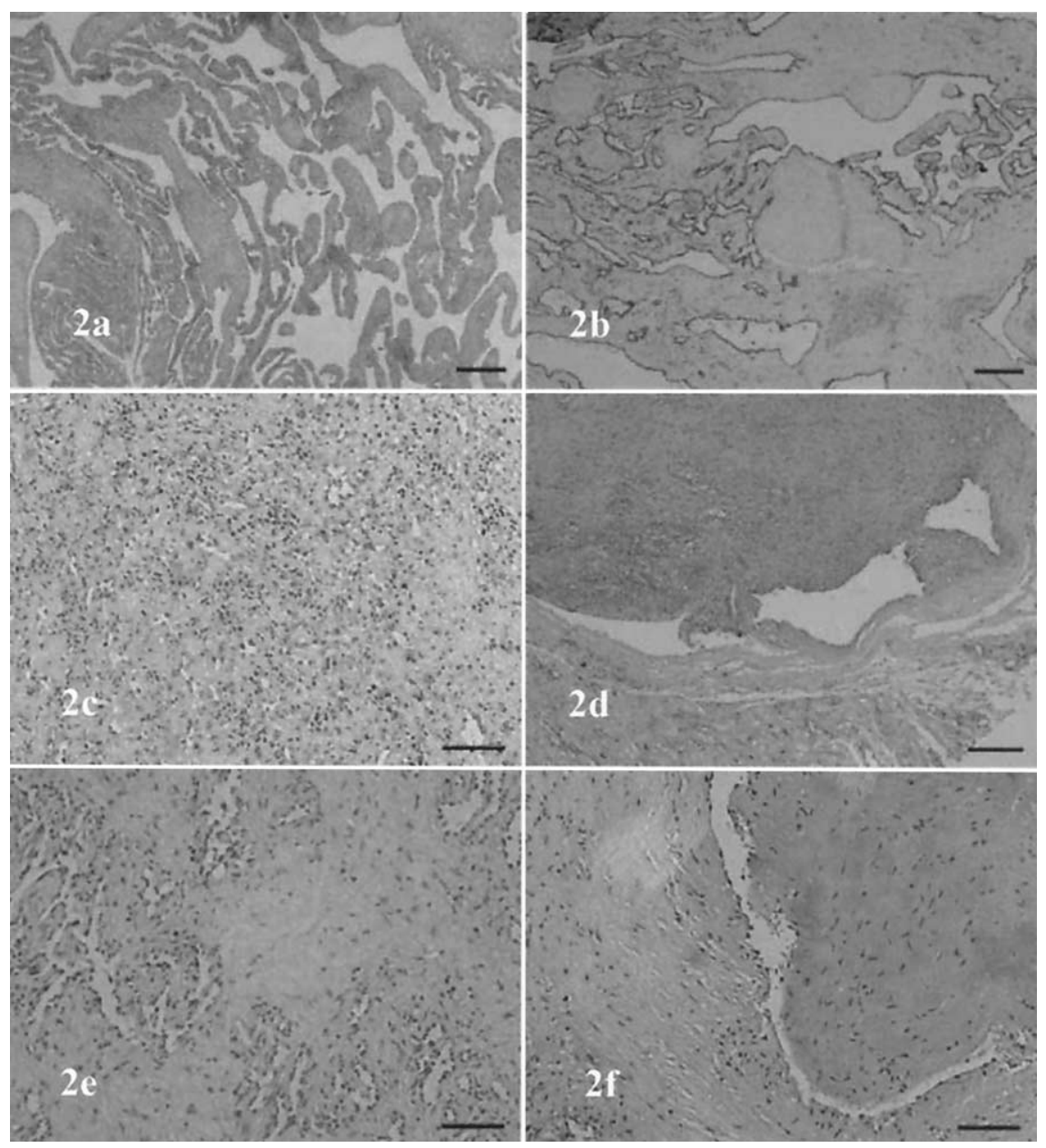

Figure 2. a, Histologic appearance of the relapsed hemangioma, with large and blood-filled wall vessels lined by endothelium. Bar is $\mathbf{4 0 0} \mu \mathrm{m}$. b, On immunohistochemical analysis, endothelial cells are strongly positive for CD31. Bar is $\mathbf{2 0 0} \mu \mathrm{m}$. c, Capillary type pattern with small vascular spaces and mild interstitial fibrosis. Bar is $\mathbf{1 0 0} \boldsymbol{\mu \mathrm { m }}$. d, Basal zone with endocardium and subendocardial myocardium. Bar is $100 \mu \mathrm{m}$. e, Interstitial hyalinizing sclerosis. Bar is $\mathbf{5 0} \mu \mathrm{m}$. f, Myxoid interstitial tissue. Bar is $\mathbf{1 0 0} \boldsymbol{\mu \mathrm { m }}$. Hematoxylin and eosin stain.

mamillated, and soft. Sections $5 \mu \mathrm{m}$ thick were prepared and stained with hematoxylin and eosin, periodic acid Schiff (PAS), and Weigert method for the elastic fibers, van Gieson method for the collagens, alcian PAS, and silver methanamine. Further sections were used for the immunohistochemical analysis with CD31 antibody, factor VIII-related antigen antibody, and Ki 67 antibody.

The main microscopic pattern was characterized by cavernous vascular spaces filled by endothelial cells (Figure 2, $a$ ), which reacted to both CD31 (Figure 2, $b$ ) and factor VIII-related antigen. The stroma was mainly fibrous with focal areas of myxoid tissue (Figure 2,f). The minor histologic pattern was characterized by little areas with lobular arrangement of multiple tightly packed capillaries (Figure 2,c). In these nodules, zones of hyalinizing tissue could be seen (Figure 2,e).

At 6 months the patient was alive but the occurrence of a complete atrioventricular block required the implantation of a bicameral pacemaker.

\section{Discussion}

Hemangiomas are vascular tumors composed of blood vessels that can be either capillaries or large cavernous vascular channels. Cardiac hemangiomas may result in compression of cardiac structures, outflow tract obstruction, pericardial effusions, and congestive heart failure. They represent a rare primary benign cardiac tumor. The widespread use of noninvasive imaging techniques has permitted their early detection. Cardiac hemangiomas have an unpredictable outcome and may involute, stop growing, or proliferate indefinitely. ${ }^{4}$ Complete surgical resection is considered curative and normally results in cessation of symptoms. Intracavitary and intramyocardial hemangiomas have a potential risk of midterm recurrence, especially if there has been an incomplete surgical resection. $^{5}$

To the best of our knowledge, this is the first report of a late recurrence of cardiac hemangioma in the same cardiac chamber location. 
The superficiality of the implant base and the late appearance of the recurrent neoplasia could rule out the hypothesis of an incomplete resection of the original tumor, raising the question about an unexplained potential growth from a second "pretumorous" metasynchronous cellular focus. To our knowledge, this issue is not supported by the literature to date. All the echocardiographic tests performed in the 10-year follow-up from the first operation showed negative results, confirming the recent growth of the recurrence.

We conclude that it is impossible to exclude a late neoplastic recurrence after resection of a cardiac hemangioma. The causes of recurrence are not completely known. A long-term echocardiographic follow-up is mandatory to monitor for representation of hemangiomas.

\section{References}

1. Straus R, Merliss R. Primary tumors of the heart. Arch Pathol. 1945; 39:74-8.

2. Brizard C, Latremouille C, Jebara VA, et al. Cardiac hemangiomas. Ann Thorac Surg. 1993;56:390-4.

3. Van Trigt P III, Sabiston DC Jr. Tumors of the heart. In: Sabiston DC, Spencer FC. 6th ed. Philadelphia: WB Saunders; 1995. Surgery of the chest, Vol 2:p. 2069-86.

4. Abad C, Campo E, Estruch R, et al. Cardiac hemangioma with papillary endothelial hyperplasia: report of resected case and review of literature. Ann Thorac Surg. 1990;49:305-8.

5. Scully RE, Mark EJ, McNeely BU. Case records of the Massachusetts General Hospital. Case 4-1983. N Engl J Med. 1983;308:20614.

\title{
Successful treatment of a mycotic aortic arch aneurysm associated with an isolated left vertebral artery
}

\author{
Yuji Naito, MD, Masato Nakajima, MD, Hidenori Inoue, MD, Eiki Mizutani, MD, and Koji Tsuchiya, MD, Kofu-shi, Japan
}

M ycotic aneurysms are rare in this age of antibiotics, but they are life-threatening. The isolated left vertebral artery (ILVA) is a left vertebral artery originating directly from the aortic arch. It is one of the most common aortic arch branch anomalies, occurring in about $4 \%$ of the general population. The case report presented here is of a mycotic aortic arch aneurysm associated with an ILVA.

\section{Clinical Summary}

A 65-year-old man was admitted to another hospital for investigation into back pain that had lasted for a few hours and disappeared spontaneously. He had reported a cough and febrile illness about 2 months earlier. He was not receiving any drugs, and his medical history was unremarkable. Chest computed tomographic findings led to a diagnosis of aortic arch aneurysm, and the patient was referred to us for surgical treatment. The patient was afebrile on admission. Vital signs were normal, as were cardiopulmonary sounds. Cardiovascular examination revealed a good pulse without bruits at all sites. Laboratory tests showed a leukocyte count of $9500 \times 10^{9}$ cells/L and a C-reactive protein level of $7.62 \mathrm{mg} / \mathrm{dL}$.

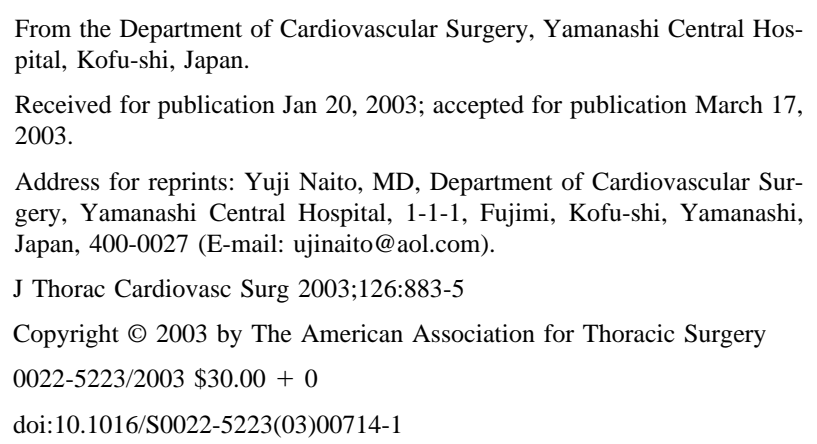

Chest radiography revealed a mildly enlarged mediastinal shadow and normal cardiothoracic ratio. Chest computed tomographic scan (Figure 1, $A$ and $B$ ) confirmed a multisaccular aneurysm of the aortic arch, and both magnetic resonance angiography (Figure 2) and digital subtraction angiography (Figure 1,B) depicted an ILVA arising from the aneurysmal wall.

We performed semiurgent surgery because the patient was at risk for rupture of the aneurysm. During surgery, dense adhesion around the aortic arch vessels was noted, probably caused by infection-induced inflammation. Total arch replacement with a 4-branched collagen-pretreated aortic arch graft (Hemashield Branched Graft; Meadox Medical, Oakland, NJ) was completed with selective cerebral perfusion. Cardiopulmonary bypass was established with ascending aortic and single right atrial cannulation. Under a condition of circulatory arrest at a rectal temperature of $28^{\circ} \mathrm{C}$, the aneurysm was opened, then selective cerebral perfusion was started through the innominate and left common carotid arteries. The aneurysmal wall was thickened and edematous, with some atherosclerotic lesions containing druses. The left subclavian artery (LSA) and ILVA were simply clamped. After careful inspection of the aorta, dissection was extended for utmost eradication of infected tissue toward healthy-looking aorta, where distal anastomosis was completed. After proximal graft anastomosis and LSA repair were completed, the shortened ILVA was reconstructed with a saphenous vein graft interposed between the native ILVA and the side of the graft branch anastomosed to the LSA. After all cervical vessels were reconstructed, cardiopulmonary bypass was stopped. The cardiopulmonary bypass, selective cerebral perfusion, circulatory arrest, and aortic crossclamping times were 164, 97, 49, and 82 minutes, respectively. Streptococcus pneumoniae, susceptible to cefazolin and to meropenem, was cultured from aortic lesion tissue. The patient was treated intravenously with $1 \mathrm{~g}$ cefazolin during surgery and $2 \mathrm{~g}$ meropenem after surgery. After 2 weeks of antibiotic therapy, he was discharged on 